\title{
Analysis on risk factors of power grid planning under the background of electric power system reform in China-An Interpretative Structural Modeling
}

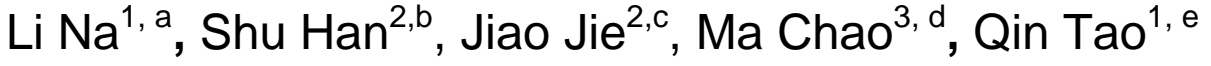 \\ ${ }^{1}$ State Grid Tianjin Economic Research Institute \\ Tianjin, China \\ ${ }^{2}$ North China Electric Power University \\ School of Economics and Management \\ Beijing, China \\ ${ }^{3}$ State Grid Tianjin Electric Power Company \\ Tianjin, China \\ alinajamie@163.com, ${ }^{b}$ tmkad2014@sina.com, ${ }^{c}$ jiaojie_ncepu@163.com, ${ }^{d}$ tjdlmc@sina.com, \\ e sniper423@qq.com
}

Keywords: Electric power system reform; Power grid planning; Risk factor; Interpretative structural modeling

Abstract. With the deepening of China's electric power system reform, the environment of power grid planning will be more complex. In this paper, the risk factors that may affect the power grid planning are identified by the Delphi method from three aspects: the generation side, the transmission and distribution side and the sale side; Then, the correlation matrix within the influencing factors is constructed by using the interpretative structural modeling. In the end, the reachable matrix of risk factors is obtained by MATLAB. And we get 5 typical risk factors transfer chain, finally.

\section{Introduction}

A new round power system reform has made a clear development direction of the electricity industry, which will break the vertical integration industry structure to build an efficient competitive power market system ${ }^{[1]}$. These will lead the future power grid planning work to face more uncertainty factors, and the formulation of the planning scheme will be influenced by a more open market environment ${ }^{[2]}$. Finding out new factors that affect the planning scheme in the future, and studying the risk transfer chain after the reform of power system is helpful to explore the optimization method of power network planning and decision-making under the new situation ${ }^{[3-4]}$. In the meantime, it is helpful to strengthen the lean management level of power grid assets and incremental assets, and ensure the sustainable development of the power grid.

The Interpretative structural modeling is a tool that can be used to analyze the relationship among risk factors and it can also display how risk factors play work in different transfer chains ${ }^{[5-6]}$. Thus, the paper applied the ISM model to risk factors analysis which have an effect on power grid planning.

\section{Interpretative Structural Modeling}

Interpretive Structural Modeling (ISM) is characterized by that the complex system is decomposed into several subsystems. Using the people's practical experience and knowledge, as well as the help of the computer, the system will be constructed as a multi-layer hierarchical interpretation structure mode ${ }^{[7-8]}$. Steps of constructing the ISM are as follows:

Addressing system factor set

Sorting out system composition factors to determine the set of system factors, as follows:

$$
N=\left\{r_{i} \mid(i=1,2, \mathrm{~L}, n)\right\}
$$




\section{The formation of awareness model}

Determine whether there is a direct binary relationship between any two factors of the factor set. The direct binary relationship between any two factors $A$ and $B$ can be expressed as:

$$
a_{i j}= \begin{cases}0 & (\text { A has no effect on B }) \\ 1 & (\text { A has an effect on B })\end{cases}
$$

Generating an adjacency matrix

The sum of all direct binary relations constitutes an adjacency matrix as:

$$
A=\left(a_{i j}\right)_{n \times n}
$$

Generating a reachable matrix

The reachability matrix is calculated according to the transfer function. The calculation formula is as following:

$$
(A+I) \neq(A+I)^{2} \neq \mathrm{L} \neq(A+I)^{m}=(A+I)^{m+1}=M
$$

Where the matrix multiplication satisfies the Boolean algebra algorithm and $I$ is the identity matrix. The essence of this algorithm is to add $A$ to $I$ and then multiply by the Boolean algebraic algorithm until all products are equal after a certain power operation. The result is the reachability matrix.

\section{Class division}

Class division is to divide different factors into different levels. The set affected by factor $r_{i}$ is defined as the reachable set $P\left(r_{i}\right)$ of factor $r_{i}$, The factor set of influencing factor $r_{i}$ is defined as the prior set $Q\left(r_{i}\right)$ of factor $r_{i}$. If $P\left(r_{i}\right) \cap Q\left(r_{i}\right)=P\left(r_{i}\right)$, then $r_{i}$ is the highest factor, that is, the factor satisfying the condition is the same class $L_{1}$. And so on, we can get different classes $L_{2}, L_{3} \ldots$

\section{Generating a hierarchical diagram}

At the end of the class division, the hierarchical structure of the system is represented as a directed graph.

Generating risk transfer chains

According to the hierarchical structure, the risk transfer chain is obtained.

\section{Identifications of Influencing Factors of electric power system reform on power system planning}

Compared with the situation before the reform, the power industry in the hair, transmission, distribution, sale of several links have undergone great changes. Affected by these changes, the uncertain factors of the power grid planning scheme will increase. This section is mainly to determine the risk factors of power grid planning on Delphi from the power generation side, transmission and distribution side and the electricity sales side. As a result, we get 18 major risk factors that affect the power grid planning program. In the power generation side, the risk factors are as followings: (1) the risk of new energy power generation scale; (2) the risk of distributed power access scale; (3) the risk of coordinating with power sources planning; (4) the fluctuation risk of various kinds of on-grid electricity price; (5) the fluctuation risk of subsidy on renewable energy price. In the transmission and distribution side, the risk factors are as followings: (6) the fluctuation risk of transmission and distribution price; (7) the fluctuation risk of integrated line loss rate; (8) power grid reliability risk; (9) the risk of coordinating the main network planning and distribution planning;(10) the scale risk of incremental distribution grid. In the electricity sales side, the risk factors are as followings: (11) the accuracy rate risk of various types of load forecasting; (12) the risk of various kinds of load fluctuations in electricity consumption; (13) the volatility risk of various types of sales price; (14) the risk of Large Consumers Direct-purchasing; (15) the risk of diversified retail electricity suppliers. Finally, there are some other risk factors: (16) the risk of substation location and line corridor change; (17) the risk of design scheme change; (18) the policy risk leaded by power system reform. 


\section{Hierarchical Structure and Transfer Chains of Risk Factors based on Interpretative Structural Modeling}

First of all, the paper determined the direct impact of each factor on the other factors. Based on these impacts, the directed cross-correlation and the awareness model among the factors can be established, which is the basis to establish the adjacency matrix for the next step. Then, according to the adjacency matrix, we can obtain the reachable matrix for risk factors by the Boolean operation. In this step, it maybe use the MATLAB software. Finally, through the principle of the hierarchical division, influence factors can be stratified and transfer chains of risk factors can be finally obtained. Table 1 displays the directed cross-correlation for each factor.

Table 1 The Influence Relationships among Risk Factors of Power Network Planning

\begin{tabular}{|c|c|c|c|}
\hline Factors & $\begin{array}{c}\text { Factors that are } \\
\text { directly affected }\end{array}$ & Factors & $\begin{array}{c}\text { Factors that are directly } \\
\text { affected }\end{array}$ \\
\hline 1 & 3,8 & 10 & $7,8,9,16$ \\
\hline 2 & $3,7,8,11$ & 11 & 16 \\
\hline 3 & 16,17 & 12 & 16,17 \\
\hline 4 & $1,12,13,14$ & 13 & $12,14,15$ \\
\hline 5 & 1,4 & 14 & $7,9,10$ \\
\hline 6 & 14,15 & 15 & - \\
\hline 7 & 16 & 16 & - \\
\hline 8 & 16,17 & 17 & 4,10 \\
\hline 9 & 16,17 & 18 & $4,6,10,13,14,15$ \\
\hline
\end{tabular}

According to Table 1, the direct relationships among 18 risk factors can form the adjacency matrix $A_{\mathrm{ij}}$ as shown in Figure 1 in which the first row and the first column are serial numbers of risk factors. The number 1 in the adjacency matrix $A_{\mathrm{ij}}$ indicates that the risk factor of the line $i$ have a direct impact on the risk factor of the column $j$, while the number 0 means that there is no obvious correlativity between them.

On the basis of the elements and their mutual relations in the adjacency matrix $A_{\mathrm{ij}}$, the paper uses the software MATLAB to do the Boolean operation on the matrix $A_{\mathrm{ij}}$. Figure 2 displays the reachability matrix for these risk factors.

\begin{tabular}{|c|c|c|c|c|c|c|c|c|c|c|c|c|c|c|c|c|c|c|}
\hline $\mathbf{A}_{\mathbf{j}}$ & 1 & 2 & 3 & $\mathbf{4}$ & 5 & 6 & 7 & $\mathbf{8}$ & $\mathbf{9}$ & $\mathbf{1 0}$ & $\mathbf{1 1}$ & $\mathbf{1 2}$ & $\mathbf{1 3}$ & $\mathbf{1 4}$ & $\mathbf{1 5}$ & 16 & 17 & 18 \\
\hline 1 & 0 & 0 & 1 & 0 & 0 & 0 & 0 & 1 & 0 & 0 & 0 & 0 & 0 & 0 & 0 & 0 & 0 & 0 \\
\hline 2 & 0 & 0 & 1 & 0 & 0 & 0 & 1 & 1 & 0 & 0 & 1 & 0 & 0 & 0 & 0 & 0 & 0 & 0 \\
\hline 3 & 0 & 0 & 0 & 0 & 0 & 0 & 0 & 0 & 0 & 0 & 0 & 0 & 0 & 0 & 0 & 1 & 1 & 0 \\
\hline 4 & 1 & 0 & 0 & 0 & 0 & 0 & 0 & 0 & 0 & 0 & 0 & 1 & 1 & 1 & 0 & 0 & 0 & 0 \\
\hline 5 & 1 & 0 & 0 & 1 & 0 & 0 & 0 & 0 & 0 & 0 & 0 & 0 & 0 & 0 & 0 & 0 & 0 & 0 \\
\hline 6 & 0 & 0 & 0 & 0 & 0 & 0 & 0 & 0 & 0 & 0 & 0 & 0 & 0 & 1 & 1 & 0 & 0 & 0 \\
\hline 7 & 0 & 0 & 0 & 0 & 0 & 0 & 0 & 0 & 0 & 0 & 0 & 0 & 0 & 0 & 0 & 1 & 0 & 0 \\
\hline 8 & 0 & 0 & 0 & 0 & 0 & 0 & 0 & 0 & 0 & 0 & 0 & 0 & 0 & 0 & 0 & 1 & 1 & 0 \\
\hline 9 & 0 & 0 & 0 & 0 & 0 & 0 & 0 & 0 & 0 & 0 & 0 & 0 & 0 & 0 & 0 & 1 & 1 & 0 \\
\hline 10 & 0 & 0 & 0 & 0 & 0 & 0 & 1 & 1 & 1 & 0 & 0 & 0 & 0 & 0 & 0 & 1 & 0 & 0 \\
\hline 11 & 0 & 0 & 0 & 0 & 0 & 0 & 0 & 0 & 0 & 0 & 0 & 0 & 0 & 0 & 0 & 1 & 0 & 0 \\
\hline 12 & 0 & 0 & 0 & 0 & 0 & 0 & 0 & 0 & 0 & 0 & 0 & 0 & 0 & 0 & 0 & 1 & 1 & 0 \\
\hline 13 & 0 & 0 & 0 & 0 & 0 & 0 & 0 & 0 & 0 & 0 & 0 & 1 & 0 & 1 & 1 & 0 & 0 & 0 \\
\hline 14 & 0 & 0 & 0 & 0 & 0 & 0 & 1 & 0 & 1 & 1 & 0 & 0 & 0 & 0 & 0 & 0 & 0 & 0 \\
\hline 15 & 0 & 0 & 0 & 0 & 0 & 0 & 0 & 1 & 0 & 1 & 0 & 0 & 0 & 0 & 0 & 0 & 0 & 0 \\
\hline 16 & 0 & 0 & 0 & 0 & 0 & 0 & 0 & 0 & 0 & 0 & 0 & 0 & 0 & 0 & 0 & 0 & 0 & 0 \\
\hline 17 & 0 & 0 & 0 & 0 & 0 & 0 & 0 & 0 & 0 & 0 & 0 & 0 & 0 & 0 & 0 & 0 & 0 & 0 \\
\hline 18 & 0 & 0 & 0 & 1 & 1 & 1 & 0 & 0 & 0 & 1 & 0 & 0 & 1 & 1 & 1 & 0 & 0 & 0 \\
\hline
\end{tabular}

\begin{tabular}{|c|c|c|c|c|c|c|c|c|c|c|c|c|c|c|c|c|c|c|}
\hline & 16 & 17 & 3 & 7 & 8 & 9 & 11 & 1 & 2 & 10 & 12 & 14 & 15 & 4 & 5 & 6 & 13 & 18 \\
\hline 16 & 1 & 0 & 0 & 0 & 0 & 0 & 0 & 0 & 0 & 0 & 0 & 0 & 0 & 0 & 0 & 0 & 0 & 0 \\
\hline 17 & 0 & 1 & 0 & 0 & 0 & 0 & 0 & 0 & 0 & 0 & 0 & 0 & 0 & 0 & 0 & 0 & 0 & 0 \\
\hline 3 & 1 & 1 & 1 & 0 & 0 & 0 & 0 & 0 & 0 & 0 & 0 & 0 & 0 & 0 & 0 & 0 & 0 & 0 \\
\hline 7 & 1 & 0 & 0 & 1 & 0 & 0 & 0 & 0 & 0 & 0 & 0 & 0 & 0 & 0 & 0 & 0 & 0 & 0 \\
\hline 8 & 1 & 1 & 0 & 0 & 1 & 0 & 0 & 0 & 0 & 0 & 0 & 0 & 0 & 0 & 0 & 0 & 0 & 0 \\
\hline 9 & 1 & 1 & 0 & 0 & 0 & 1 & 0 & 0 & 0 & 0 & 0 & 0 & 0 & 0 & 0 & 0 & 0 & 0 \\
\hline 11 & 1 & 0 & 0 & 0 & 0 & 0 & 1 & 0 & 0 & 0 & 0 & 0 & 0 & 0 & 0 & 0 & 0 & 0 \\
\hline 1 & 1 & 1 & 1 & 0 & 1 & 0 & 0 & 1 & 0 & 0 & 0 & 0 & 0 & 0 & 0 & 0 & 0 & 0 \\
\hline 2 & 1 & 1 & 0 & 1 & 1 & 0 & 1 & 0 & 1 & 0 & 0 & 0 & 0 & 0 & 0 & 0 & 0 & 0 \\
\hline 10 & 1 & 1 & 0 & 1 & 1 & 1 & 0 & 0 & 0 & 1 & 0 & 0 & 0 & 0 & 0 & 0 & 0 & 0 \\
\hline 12 & 1 & 1 & 0 & 0 & 0 & 0 & 0 & 0 & 0 & 0 & 1 & 0 & 0 & 0 & 0 & 0 & 0 & 0 \\
\hline 14 & 1 & 1 & 0 & 1 & 0 & 1 & 0 & 0 & 0 & 0 & 0 & 1 & 0 & 0 & 0 & 0 & 0 & 0 \\
\hline 15 & 0 & 0 & 0 & 0 & 1 & 0 & 0 & 0 & 0 & 1 & 0 & 0 & 1 & 0 & 0 & 0 & 0 & 0 \\
\hline 4 & 1 & 1 & 0 & 0 & 0 & 0 & 0 & 1 & 0 & 0 & 1 & 1 & 0 & 1 & 0 & 0 & 0 & 0 \\
\hline 5 & 1 & 1 & 0 & 0 & 0 & 0 & 0 & 1 & 0 & 0 & 0 & 0 & 0 & 1 & 1 & 0 & 0 & 0 \\
\hline 6 & 1 & 1 & 0 & 0 & 0 & 0 & 0 & 0 & 0 & 0 & 0 & 1 & 1 & 0 & 0 & 1 & 0 & 0 \\
\hline 13 & 1 & 1 & 0 & 0 & 0 & 0 & 0 & 0 & 0 & 0 & 0 & 1 & 1 & 0 & 0 & 0 & 1 & 0 \\
\hline 18 & 1 & 1 & 1 & 1 & 1 & 1 & 1 & 1 & 1 & 1 & 0 & 1 & 1 & 1 & 0 & 1 & 1 & 1 \\
\hline
\end{tabular}

Figure 1 The Adjacency Matrix of Risk

Factors For Power Grid Planning
Figure 2 The Reachability Matrix of Risks Factors in Power in Power Grid Planning

According to the hierarchical principle in the ISM modeling, the risk factors that affect the power network planning in the matrix can be divided into 6 levels. The hierarchical structures for these risk 
factors are as followings: $\mathrm{L} 1=\{16,17\} ; \mathrm{L} 2=\{3,7,8,9,11\} ; \mathrm{L} 3=\{1,2,10\} ; \mathrm{L} 4=\{12,14,15\}$; $\mathrm{L} 5=\{4,5,6,13\} ; \mathrm{L} 6=\{18\}$;

What's more, it can be seen that Figure 2 describes the mutual influence relationship of each layer. Based on it, the hierarchical structure diagram of risk factors for power network planning can be drawn as shown in Figure 3.

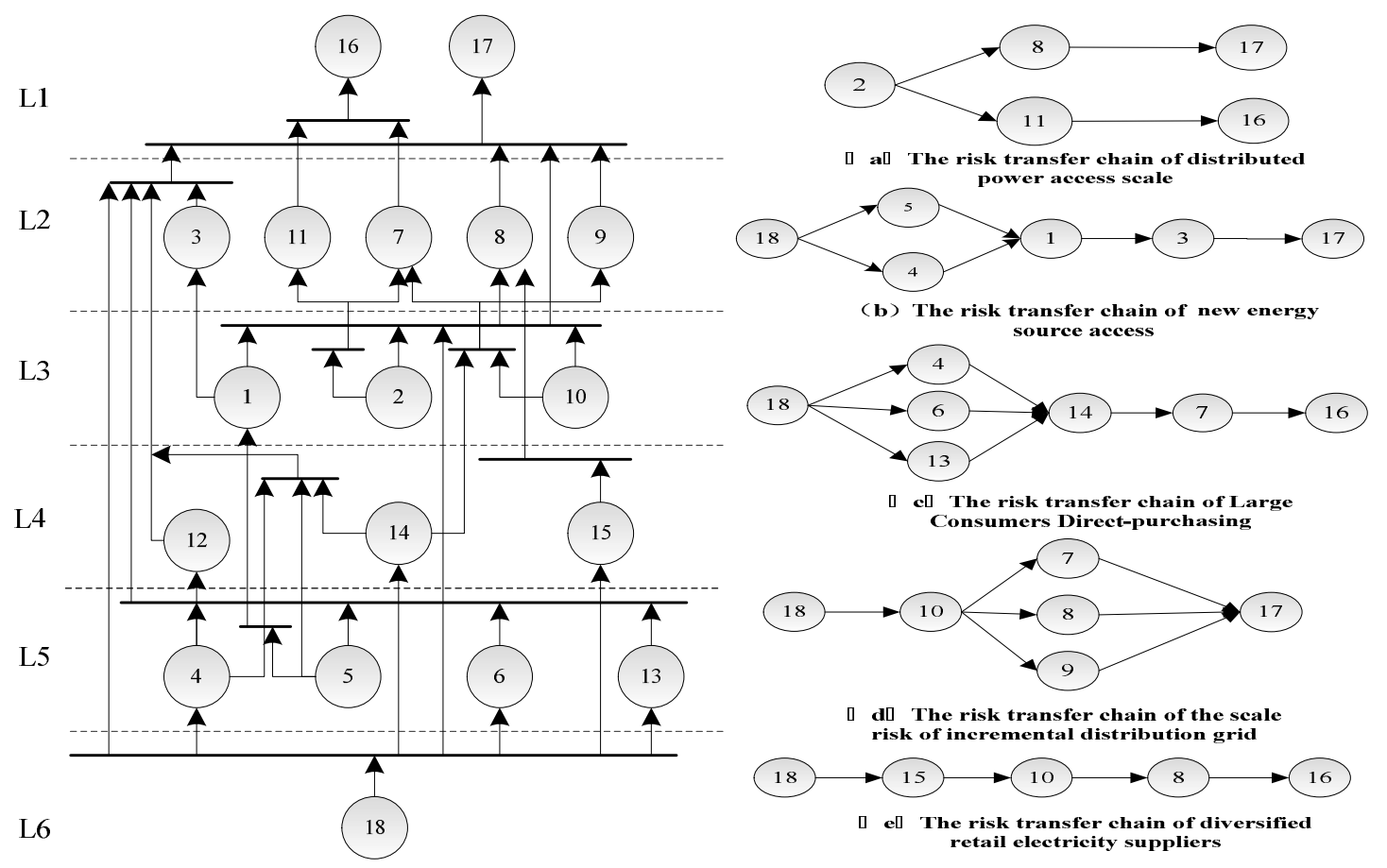

Figure 3 The Hierarchical Structure Diagram for

Risk Factors in Power Grid Planning

Figure 4 Figure Five Typical Risk Transfer Chains

In the diagram, different layer represents the different effect degree of the 18 risk factors. These 18 risk factors in these 6 layers form different risk transfer chains from bottom to up. And the lower the layer is, the greater the effect degree is of it. After analyzing the above hierarchical structure diagram, five typical power transfer chains can be selected from the figure 3 as shown in Figure 4.

\section{Conclusion}

Through the above research, we can get the following results:

(1) The access of distributed generation impacts the power grid planning scheme through affecting the power grid reliability and the accuracy of electric power load forecasting.

(2) In the risk transfer chain of new energy source access, the electricity power system reform policies affect the various types feed-in tariffs and renewable electricity price subsidies. And the results will influence the coordination between power grid planning and power sources planning, which will eventually affect the power grid planning scheme.

(3) The electricity power system reform policies affect various types feed-in tariffs, transmission and distribution price, as well as various types sales price, which will affect the size of Large Consumers Direct-purchasing. Then, the scale of Large Consumers Direct-purchasing will affect the integrated line loss rate that finally influences the power grid planning scheme.

(4) Firstly, the electricity power system reform policies will affect the scale of incremental distribution grid which will have an effect on the integrated line loss rate and the coordination between the main network planning and distribution planning. Finally, the power grid planning scheme maybe be influenced by them.

(5) In the risk transfer chain of diversified retail electricity suppliers, the electricity power system reform policies will affect the amount of diversified retail electricity suppliers, firstly. Then, the amount of diversified retail electricity suppliers has an effect on the scale of incremental distribution 
grid, which will affect the power grid reliability and the coordination between the main network planning and distribution planning. Finally, the power grid planning scheme maybe be influenced.

\section{Acknowledgment}

The work described in this paper was supported by research grant from science and technology projects of State Grid Tianjin Electric Power Company in China.

\section{References}

[1] Chinese Journal of Chemical Engineering,vol.23,pp.2029-2036,2015. BAI Mei, "The target, difficulty and route choice of the new round power system reform" Price:Theory\&Practice, vol.7, pp. 10-15, 2014.

[2] SHEN Hongyu, CHEN Jin, GUI Sanrong, "Influence and Countermeasure of Distribution Network Planning for Power Grid Enterprises under Electric Reform" Electric Power Construction, Vol. 3, pp.47-51, 2016.

[3] Wang Yi Cheng Haozhong, "Review of Optimal Transmission Expansion Planning in Market Environment" Transactions of China Electrotechnical Society, vol.22, pp. 174-183, 2007.

[4] Yu Huili, "Comprehensive Evaluation and Decision-making for Transmission Network Planning Alternatives Under Risk Factors”. Northeast Electric Power University, 2014.

[5] HAN Jin-shan, TAN Zhong-fu, LIU Yan, "Research on Interpratative Structure Modeling for Risks of Electricity Retail Market” Power System Technology,vol.29,pp.14-19 2005.

[6] XU Min jie, HU Zhao guang, SHAN Bao-guo, "Analysis on influencing factors of electricity demand by using interpretative structural modeling" Electric Power,vol.42,pp.1-5,2009.

[7] Zhang Can, Sun Lei, Wen Fushuan, "An interpretative structural modeling based network reconfiguration strategy for power systems" International Journal of Electrical Power \& Energy Systems,vol.65,pp.83-93,2015.

[8] Han Yongming, Geng Zhiqiang, Zhu Qunxiong, Lin Xiaoyong," Energy consumption hierarchical analysis based on interpretative structural model for ethylene production" Chinese Journal of Chemical Engineering,vol.23,pp.2029-2036,2015. 